\title{
Imaging of 6.7-GHz Methanol Maser Sources with the Japanese VLBI Network
}

\author{
Satoko Sawada-Satoh ${ }^{* \dagger}$ \\ Yamaguchi University \\ E-mail: ssseyamaguchi-u.ac.jp
}

\begin{abstract}
We report the recent VLBI imaging of the $5_{1} \rightarrow 6_{0} A^{+}$transition $6.7 \mathrm{GHz}$ methanol maser sources associated with massive star-forming regions using the Japanese VLBI Network (JVN). Currently, JVN consists of ten antennas spread across Japan from Hokkaido to Ishigaki islands, and operates at frequencies around 6.7, 8.3 and $22 \mathrm{GHz}$. The observing system of $6.7 \mathrm{GHz}$ now has been installed in six of them; all of four NAOJ VERA stations (Mizusawa, Ogasawara, Iriki, Ishigaki), Yamaguchi 32-m antenna and ISAS Usuda 64-m antenna, with baseline lengths range from 293 to $2270 \mathrm{~km}$. We have carried out the JVN observations toward $6.7 \mathrm{GHz}$ methanol maser sources since 2005, and continue to obtain high angular resolution images of them. In this paper, we present detailed $6.7 \mathrm{GHz}$ methanol maser distributions of three sources, W3(OH), G9.62+0.20 and S269, and describe the results of each individual source.
\end{abstract}

The 9th European VLBI Network Symposium on The role of VLBI in the Golden Age for Radio Astronomy and EVN Users Meeting

September 23-26, 2008

Bologna, Italy

* Speaker.

${ }^{\dagger}$ A footnote may follow. 

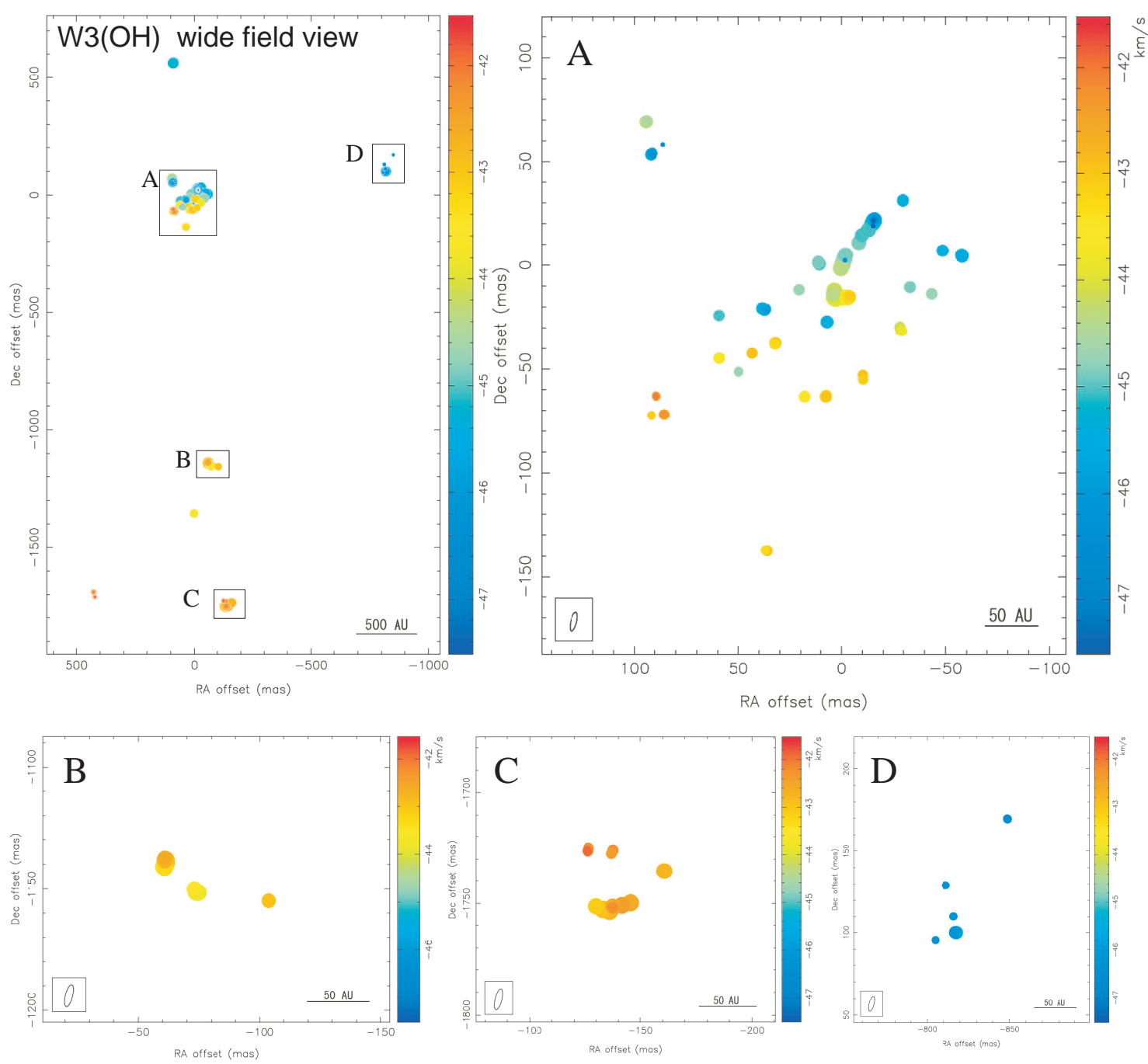

Figure 1: Wide field view of $6.7 \mathrm{GHz}$ methanol maser distribution in $\mathrm{W} 3(\mathrm{OH})$ (top-left) and close-up image for each clump (A, B, C, D).

\section{Results}

$\mathrm{W} 3(\mathrm{OH})$ is one of the most intensively studied star-forming regions at a distance of $2.0 \mathrm{kpc}$ (Hachisuka et al. 2006, Xu et al. 2006) and contains several high- and intermediate-mass young stars and protostars at different evolutionary stages. The $6.7 \mathrm{GHz}$ methanol masers are found in several clumps (Figure 1), and distribution of the maser spots agree well with the previous VLBI images by Menten et al. (1992) and Sugiyama et al. (2008). It implies that the $6.7 \mathrm{GHz}$ methanol maser distribution in $\mathrm{W} 3(\mathrm{OH})$ has been remarkably stable for $\sim 15$ years from 1992 to 2006 . We note that $22 \mathrm{GHz}$ water masers in $\mathrm{W} 3(\mathrm{OH})$ show a rapid time variability within a few years in contrast with methanol masers (e.g. Xu et al. 2000, Hachisuka et al. 2006).

G9.62+0.20 is a UC HII complex, whose two regions are associated with methanol maser emission. The $6.7 \mathrm{GHz}$ maser in G9.62+0.20 is known as the most powerful methanol maser, and it is organized into two groups in the VLBI map (Figure 2a). The main group consists of two sub 
(a) G9.62+0.20

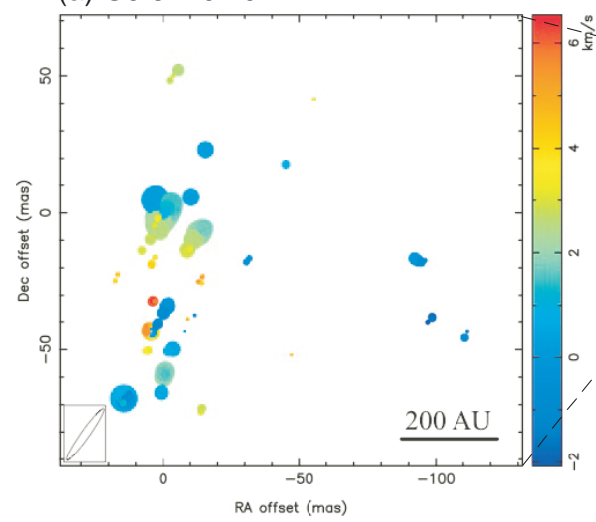

(b) S269

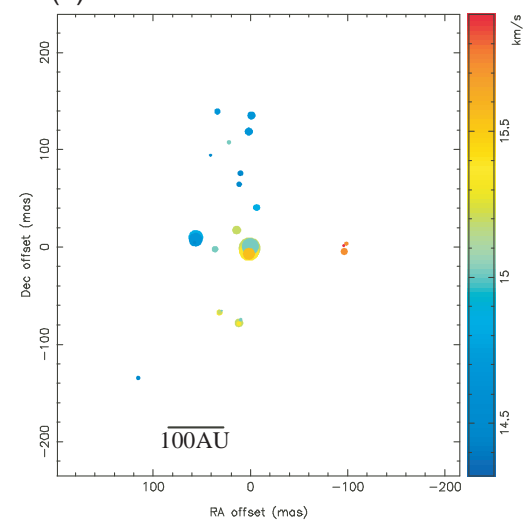

Figure 2: (a) Wide field view and close-up image of $6.7 \mathrm{GHz}$ methanol maser distribution in G9.62+0.20. (b) The distribution of $6.7 \mathrm{GHz}$ methanol masers in S269.

groups, and the maser distribution is similar to the $12 \mathrm{GHz}$ VLBI maps by Minier et al. (2000) and Goedhart et al. (2005).

S269 is an HII region located in the outer Galaxy at a distance of $5.28 \mathrm{kpc}$ (Honma et al. 2007). The $6.7 \mathrm{GHz}$ methanol masers in S269 are probably associated with the young IRAS source. The spectrum of $6.7 \mathrm{GHz}$ methanol maser consists of a single feature at $\mathrm{V}_{\mathrm{LSR}}$ of $15.2 \mathrm{~km} \mathrm{~s}^{-1}$, and it is shifted from the peak of $22 \mathrm{GHz}$ water maser spectrum $\left(19.6 \mathrm{~km} \mathrm{~s}^{-1}\right)$. The main bright methanol masers distributed along the east-west direction (Figure 2b). We note that the $22 \mathrm{GHz}$ water masers in S269 also show an aligned distribution along the east-west direction on a scale of 0.4 mas (Honma et al. 2007).

\section{Acknowledgments}

We thank all the past and present JVN members for observing assistance and support. The JVN project is led by the National Astronomical Observatory of Japan, Hokkaido University, Gifu University, Yamaguchi University, and Kagoshima University, in cooperation with Geographical Survey Institute, the Japan Aerospace Exploration Agency and the National Institute of Information and Communications Technology.

\section{References}

[1] Goedhart, S., Minier, V., Gaylard, M. J., van der Walt, D. J., 2005, MNRAS, 356, 839

[2] Hachisuka, K. et al., 2006, ApJ, 645, 337

[3] Honma, M. et al., 2007, PASJ, 59, 889

[4] Menten, K. M., Reid, M. J., Pratap, P., Moran, J. M. , Wilson, T. L., 1992, ApJ, 401, L39

[5] Minier, V., Booth, R. S., Conway, J. E., 2000, A\&A, 362, 1093

[6] Sugiyama, K. et al., 2008, PASJ, 60, 23

[7] Xu, Y., Reid, M. J., Zheng, X. W. , Menten, K. M., 2006, Science, 311, 54

[8] Xu, Y., Zheng, X. W., Zhang, F. J., Yu, Z. Y., Han, P., Scalise, E., Jr., Chen, Y. J., 2000, A\&A, 364, 232 